\title{
Treatment with somatostatin analogues and PRRT in metastatic middle ear adenoma with neuroendocrine features
}

\author{
Joana Lima Ferreira ${ }^{D} 1$, Bernardo Marques ${ }^{D 2}$, C Willemien Menke-van der Houven van Oordt³, \\ Wouter W de Herder ${ }^{4}$, Tessa Brabander5 and Johannes Hofland4
}

'Department of Endocrinology, Hospital Pedro Hispano, Matosinhos Local Health Unit, Matosinhos, Portugal, 2Department of Endocrinology, Instituto Português de Oncologia de Coimbra Francisco Gentil, Coimbra, Portugal, 3Department of Medical Oncology, Amsterdam UMC, Vrije Universiteit Medical Center, Cancer Center Amsterdam Amsterdam, The Netherlands, “Department of Internal Medicine, Section of Endocrinology, ENETS Center of Excellence, Erasmus Cancer Institute, Erasmus Medical Center, Rotterdam, The Netherlands, and ${ }^{5}$ Department of Radiology \& Nuclear Medicine, ENETS Center of Excellence, Erasmus Medical Center, Rotterdam, The Netherlands

Correspondence should be addressed to J Hofland

Email

j.hofland@erasmusmc.nl

\section{Summary}

Middle ear adenomas with neuroendocrine features (ANEF) are rare, with an estimated 150 reported cases. They usually pursue an indolent clinical course. Four reported cases of middle ear ANEF with distant metastases were treated with surgery, external beam radiation therapy (EBRT) and chemotherapy. To date, no successful systemic treatment for malignant behaviour of this rare tumour has been reported. Long-acting somatostatin analogues (SSAs) and peptide receptor radionuclide therapy (PRRT) have been used in well-differentiated metastatic neuroendocrine tumours (NETs), but their use has never been described in cases of metastatic middle ear ANEF. We report two patients with grade 1 middle ear ANEF treated with surgery and EBRT. They had stable disease for several years, until clinical symptoms appeared and extensive metastases were detected on ${ }^{68} \mathrm{Ga}$-DOTA ${ }^{0}$-Tyr ${ }^{3}$-octreotate (DOTATATE) PET/CT. Treatment with long-acting SSA was started, with stable disease for 1 year. Afterwards, despite undergoing local treatments, both patients presented progressive disease. Due to high-uptake metastases at ${ }^{68} \mathrm{Ga}$-DOTATATE PET/CT, both cases underwent four cycles of PRRT with ${ }^{177}$ Lu-DOTATATE, which secured disease control and improvement of quality of life in both. Similar to other well-differentiated NETs, SSA and PRRT could constitute efficacious therapeutic options in metastatic middle ear ANEF. Its neuroendocrine differentiation, potential to metastasize and somatostatin receptor type 2 expression prompt consideration and management of this disease as a neuroendocrine neoplasm.

\section{Learning points:}

- Our cases oppose the 2017 WHO classification of middle ear adenoma with neuroendocrine features as a benign disease.

- This entity warrants long-term follow-up, as local recurrence or persistence of disease is reported in up to $18 \%$ of surgically treated patients.

- PET/CT scan with ${ }^{8} \mathrm{G}$ Ga-labelled somatostatin analogues (SSA) can be used for staging of metastatic middle ear adenoma with neuroendocrine features.

- Unlabelled SSA and peptide receptor radionuclide therapy (PRRT) with radiolabelled SSA can be the first systemic therapeutic options for patients with advanced middle ear adenoma with neuroendocrine features. 


\section{Background}

Middle ear adenomas are rare, accounting for less than $2 \%$ of ear tumours (1) and approximately 150 cases have been reported in literature $(2,3)$. They were first described in 1976 by Hyams and Michaels and in 1980 Murphy et al. described them as having a neuroendocrine differentiation. Several denominations of this entity have been used, as there is much controversy regarding the most appropriate classification due to the heterogeneous histopathological features (3). It has been advocated that these different terms can represent different degrees of neuroendocrine differentiation of the same entity (4). According to current taxonomy, in case of neuroendocrine differentiation, it might be referred as an adenoma with neuroendocrine features (ANEF) (1). It is believed that ANEF arise from a pluripotent endodermal stem cell or from a stromal precursor derived from the neural crest and that they present dual neuroendocrine and mucin-secreting differentiation (1).

Complete tumour resection is the treatment of choice for middle ear ANEF and they usually pursue an indolent clinical course (5). The 2017 WHO classification has defined the disease as benign, without indisputable evidence of malignant potential. Nevertheless, local recurrence or persistence of disease are reported in 13-18\% of surgically treated patients (4). Regarding metastatic potential, to the best of our knowledge, only six cases of regional lymph node metastases and four cases of distant metastases have been reported $(2,5,6)$. There are no descriptions of successful systemic treatment of metastatic middle ear ANEF.

Similar considerations regarding clinical behaviour have long been advocated for gastroenteropancreatic neuroendocrine tumours (GEP-NETs). However, malignant potential is now a well-recognized universal feature (7). Systemic treatment options for well-differentiated, unresectable or metastatic GEP-NETs include longacting somatostatin analogues (SSAs) and peptide receptor radionuclide therapy (PRRT) using beta-emitting radiolabelled SSAs (8). These therapeutic modalities have never been described in cases of metastatic middle ear ANEF.

We report the first two patients with middle ear ANEF with distant metastases treated with SSAs and PRRT due to progressive disease, which led to disease control and improved quality of life.

\section{Case presentations}

A 38-year-old woman presented with persisting left facial paralysis and ear pain under analgesic medication. Cranial CT scan revealed a $20 \mathrm{~mm}$ mass in the left middle ear, suspicious of adenocarcinoma. The patient underwent a subtotal petrosectomy and pathology reported a microscopically irradical resection of a grade 1 ANEF, with extensive bone invasion. She underwent 66 Gy of adjuvant external beam radiation therapy (EBRT), and afterwards, 18Fluorodeoxyglucose PET/CT scan did not show any pathological uptake. Six years later, she was presented with extensive lymph node, liver and bone lesions on a CT scan. Liver biopsy confirmed the diagnosis of a metastatic grade 1 ANEF. ${ }^{68} \mathrm{Ga}$-DOTA ${ }^{0}-\mathrm{Tyr}^{3}$-octreotate (DOTATATE) PET/CT showed high uptake in cervical and left supraclavicular lymph nodes, multiple liver lesions and bone metastases in the ribs, spine and pelvis. The patient was started on octreotide long-acting release (LAR) $30 \mathrm{mg}$ every 4 weeks and underwent selective embolization of liver metastases. After 1 year of stable disease, she was presented with fatigue and weight loss and progression of liver and lymphatic disease was detected on CT scan. The dose of octreotide LAR $30 \mathrm{mg}$ was increased to every 2 weeks. Three months later, a ${ }^{68} \mathrm{Ga}$-DOTATATE PET/ CT revealed progression of lymphatic and liver disease (Fig. 1A and B). At this point, the patient suffered from asthenia and anorexia. It was decided to perform PRRT with four cycles of ${ }^{177} \mathrm{Lu}$-DOTATATE, resulting in a cumulative dose of $30.4 \mathrm{GBq}$. It was well tolerated, with mild gastrointestinal disturbance.

The second patient is a 57-year-old male who underwent a right subtotal petrosectomy for excision of a middle ear mass. Histopathological examination revealed a $12 \mathrm{~mm}$

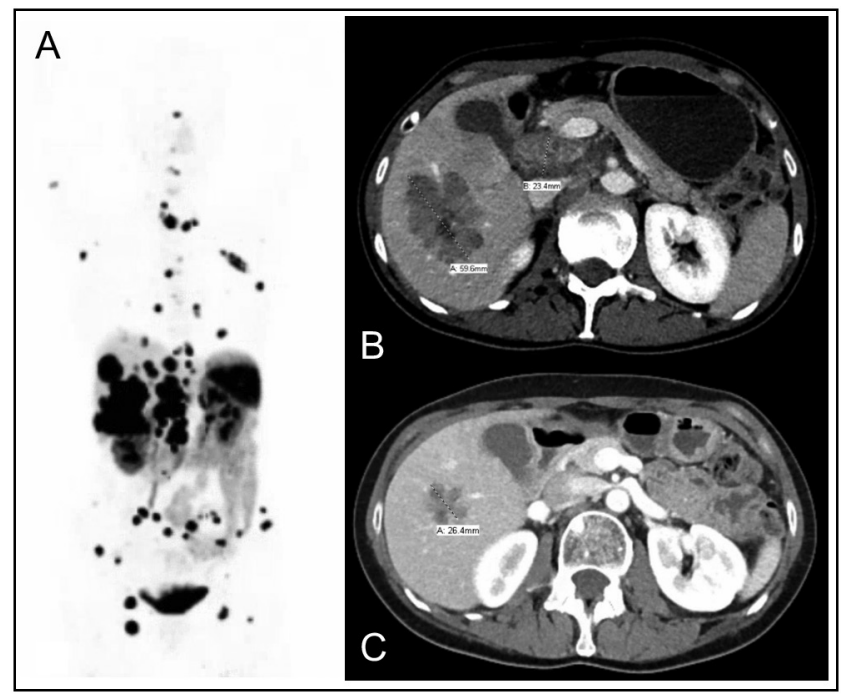

Figure 1

${ }^{68}$ Ga-DOTATATE PET/CT scan showed diffuse metastatic disease before PRRT (A). Abdominal CT scans revealed the change in liver metastases from baseline (B) to 6 months after four cycles of PRRT (C). 
grade 1 ANEF, with perivascular invasion and extension to the mastoid. Four years later, due to tumour regrowth, he underwent surgical debulking and adjuvant EBRT. Ten years later, due to back pain, a CT scan was performed, revealing extensive bone metastases to the spine and pelvis. ${ }^{68} \mathrm{Ga}$-DOTATATE PET/CT scan showed high-uptake of the lesions and bone biopsy confirmed the diagnosis of a metastatic lesion of a grade 1 ANEF. He was started on lanreotide autogel $120 \mathrm{mg}$ every 4 weeks and denosumab 120 mg every 3 months (to prevent skeletal-related events), and underwent 20 Gy EBRT from on the C7-Th2 area. One year later, he was presented with a Th6 pathological vertebral collapse with epidural protrusion and skeletal metastases in L1 and L5 and underwent palliative EBRT on the spine. A ${ }^{68} \mathrm{Ga}$-DOTATATE PET/CT scan revealed progression of disease with high uptake in cervical lymph nodes and extensive skeletal metastases (Fig. 2A and B). He underwent four cycles of PRRT (cumulative dose of 23.2 GBq). The total dose was lower than planned due to symptomatic anaemia (nadir of $4.7 \mathrm{mmol} / \mathrm{L}$ ) that required several red blood cell transfusions during treatment.

\section{Outcome and follow-up}

In the first patient, cervical, thoracic and abdominal CT performed 2 months after the last cycle of PRRT revealed a partial response of liver and lymph node metastases, while the other lesions remained stable. Six months after PRRT, her asthenia and weight were significantly improved and CT scan showed a further decrease in size of hepatic and lymph node lesions (Fig. 1C). The patient presented

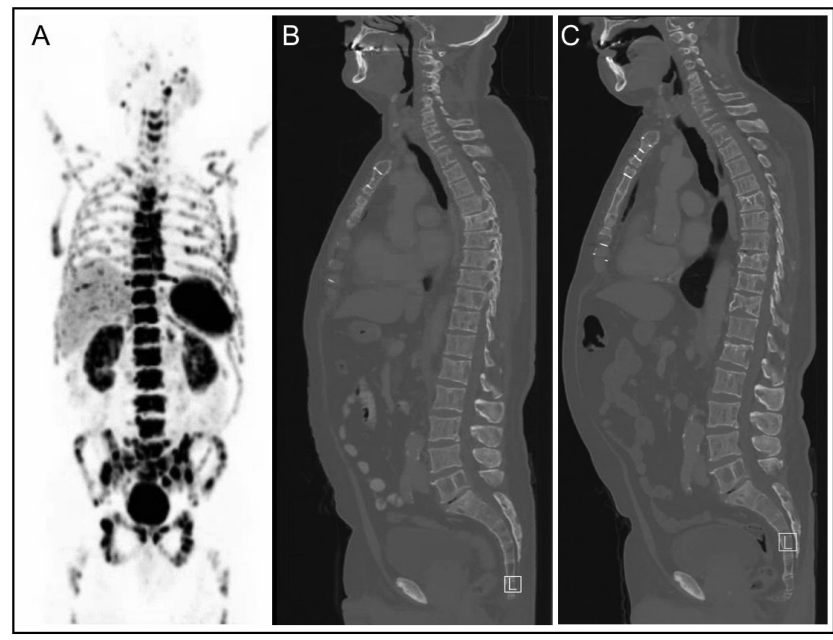

Figure 2

${ }^{68} \mathrm{Ga}$-DOTATATE PET/CT and CT scan showed extensive bone metastases before the start of PRRT (A, B). After PRRT, the CT scan revealed stable bone metastases ( $B$ and $C$ ). with constipation at 15 months after the start of PRRT and 8 months after the last cycle. Cross-sectional imaging revealed progressive disease in the liver and peritoneal carcinomatosis and she was started on chemotherapy with carboplatin and etoposide. Patient developed grade IV pancytopenia and progressive disease after three cycles of chemotherapy. Palliative care was started and she died 30 months after the start of PRRT.

After the first PRRT cycle, the second patient denied lumbar pain for the first time in 2 years. Two months after the last cycle, CT scan showed stable disease with sclerosing of bone metastases. Six months later, CT scan confirmed stable disease (Fig. 2C). The levels of haemoglobin returned to pre-treatment levels, whereas a grade 1 thrombocytopenia persisted. The patient maintains improved quality of life, with no lumbar pain, now 26 months after the start of PRRT.

\section{Discussion}

Middle ear ANEF is an uncommon disease with no known treatment option in the case of malignant spread. Up until now, only four patients have been described that developed distant metastases (2, 5, 6). Similar to our patients, the disease course from diagnosis took several years to reach metastatic stage. Besides tumour size, adjacent tissue invasion or distant metastases at diagnosis, there are no reliable markers for evaluation of middle ear ANEF aggressiveness (5). These cases oppose the WHO classification of ANEF as a benign disease and warrant further study, including long-term follow-up of patients.

These tumours have neuroendocrine differentiation and several cases have been shown to express the somatostatin receptor type 2 (SSTR2) by Somatostatin Receptor Scintigraphy $(3,5)$. Our two patients also showed ample SSTR2 expression in their metastases, with uptake on ${ }^{68} \mathrm{Ga}$-DOTATATE PET/CT clearly above that of the liver. A third case with a locally recurrent, non-metastatic middle ear ANEF also underwent a ${ }^{68} \mathrm{Ga}$-DOTATATE PET/ CT at our centre and showed positive tumour uptake, albeit lower than the liver parenchyma (data not shown). These features could have an important role in the imaging guidelines for staging in ANEF, similar to that of NETs and paragangliomas.

Treatment of previously reported metastatic cases included surgery, EBRT and chemotherapy. The only reported systemic therapy was carboplatin and VP16 chemotherapy in one case, but the patient died 5 months after the start of this therapy (5). SSTR2 expression makes middle ear ANEF 
eligible for treatment options used in other NETs, including long-acting SSAs and PRRT with radiolabelled SSAs (9). Conceptually, these SSA-guided therapeutics may constitute a better targeted approach for this well-differentiated tumour.

Somatostatin analogues have antiproliferative effects, with both PROMID and CLARINET studies showing improved time to progression and progressionfree survival (PFS) in advanced non-functioning welldifferentiated GEP-NETs treated with octreotide LAR and lanreotide, respectively. Thus, SSAs are the first-line medical treatment in GEP-NET, in which curative surgery is not feasible (8). Despite the lack of randomized clinical trials, these SSAs are often prescribed for NETs arising in other locations. The known use of SSA in middle ear ANEF is restricted to a single patient with carcinoid syndrome in an adjuvant setting (3). Our cases for the first time show that long-acting SSA can have antiproliferative effects in metastatic middle ear ANEF, leading to a progression-free interval of approximately 1 year. This is comparable to the efficacy of SSA in GEP-NETs. Given its limited toxicity and good tolerability, long-acting (unlabelled) SSA could be considered in unresectable or metastatic middle ear ANEF.

In patients with well-differentiated NET with progressive disease under SSA, PRRT with ${ }^{177} \mathrm{Lu}$-DOTATATE is a second-line treatment option, provided that there is sufficient tumour uptake on the diagnostic somatostatin receptor imaging (8). The NETTER-1 trial showed that PRRT improved PFS in progressive midgut NET compared to abovelabel dose of SSA, confirming its efficacy in somatostatin receptor-positive tumours. On top of this, PRRT is known to improve quality of life in these patients (10).

Following progression of disease during SSA treatment, PRRT was started in both patients because of the high uptake of metastases on ${ }^{68} \mathrm{Ga}$-DOTATATE PET/ CT. Besides adequate uptake on somatostatin receptor imaging, the other requirements for PRRT are sufficient bone marrow reserve, creatinine clearance above $50 \mathrm{~mL} /$ min, Karnofsky performance score above $50 \%$ and an expected survival beyond 3 months. In most cases, PRRT with ${ }^{177} \mathrm{Lu}$-DOTATATE is well tolerated. Haematological and kidney toxicity are well-known adverse events, but grade 3 and 4 toxicity occur in less than $1 \%$ of patients. Our second patient had pancytopenia due to extensive bone marrow metastases and required red cell transfusion to support his haemoglobin levels during PRRT. PRRT was halted after $23.2 \mathrm{GBq}$ due to recurrent grade 3 anaemia. In both cases, there were no other PRRT-related toxicities. In our patients, PRRT induced disease control for 15 months and at least 26 months, respectively, as well as improved their tumour-related symptoms.
In conclusion, our cases oppose the WHO classification of ANEF as a benign disease and warrant further study, including long-term follow-up. Similar to other welldifferentiated NETs, SSA and PRRT can constitute efficacious systemic therapeutic options in metastatic middle ear ANEF. Its neuroendocrine differentiation, potential to metastasize and SSTR2 expression prompt consideration and management of this disease as a type of well-differentiated NET.

\section{Declaration of interest}

Wouter de Herder is a Senior Editor of Endocrinology, Diabetes and Metabolism Case Reports. Wouter de Herder was not involved in the review or editorial process for this paper, on which he is listed as an author. The other authors declare that there is no conflict of interest that could be perceived as prejudicing the impartiality of the research reported.

\section{Funding}

This research did not receive any specific grant from any funding agency in the public, commercial or not-for-profit sector.

\section{Patient consent}

Written informed consent has been obtained from both patients for publication of the submitted article and accompanying images.

\section{Author contribution statement}

JLF and BM drafted the manuscript and contributed equitably. JH was involved in critical revision of all drafts of the manuscript. CWM, WWH, TB and $\mathrm{JH}$ were involved in patient care. All authors have approved the final version of the manuscript.

\section{Acknowledgements}

JLF and BM thank the Portuguese Society of Endocrinology, Diabetes and Metabolism for the support of the international fellowship programme in Erasmus Medical Center, Rotterdam.

\section{References}

1 El-Naggar AK. WHO Classification of Head and Neck Tumours. France: International Agency for Research on Cancer, 2017.

2 Bell D, El-Naggar AK \& Gidley PW. Middle ear adenomatous neuroendocrine tumors: a 25-year experience at MD Anderson Cancer Center. Virchows Archiv 2017471 667-672. (https://doi. org/10.1007/s00428-017-2155-6)

3 Marinelli JP, Cass SP, Mann SE, Haynes DS, Hunter JB, Isaacson B, Sweeney AD, Pelosi S, Willcox TO, O'Connell BP et al. Adenomatous neuroendocrine tumors of the middle ear: a multi-institutional investigation of 32 cases and development of a staging system. Otology and Neurotology 201839 e712-e721. (https://doi.org/10.1097/ MAO.0000000000001905) 
4 Torske KR \& Thompson LD. Adenoma versus carcinoid tumor of the middle ear: a study of 48 cases and review of the literature. Modern Pathology 200215 543-555. (https://doi.org/10.1038/ modpathol.3880561)

5 Gaafar A, Ereno C, Ignacio Lopez J, Fernandez de Larrinoa A, Grande J, Salazar J, Yarnoz J \& Bilbao FJ. Middle-ear carcinoid tumor with distant metastasis and fatal outcome. Hematology/Oncology and Stem Cell Therapy 20081 53-56. (https://doi.org/10.1016/s16583876(08)50061-6)

6 Fundakowski CE, Chapman JR \& Thomas G. Middle ear carcinoid with distant osseous metastasis. Laryngoscope 2013123 779-782. (https://doi.org/10.1002/lary.23434)

7 Hofland J, Kaltsas G \& de Herder WW. Advances in the diagnosis and management of well-differentiated neuroendocrine neoplasms. Endocrine Reviews 202041 371-403. (https://doi.org/10.1210/endrev/ bnz004)
8 Pavel M, O'Toole D, Costa F, Capdevila J, Gross D, Kianmanesh R, Krenning E, Knigge U, Salazar R, Pape UF et al. Enets consensus guidelines update for the management of distant metastatic disease of intestinal, pancreatic, bronchial neuroendocrine neoplasms (NEN) and NEN of unknown primary site. Neuroendocrinology 2016103 172-185. (https://doi.org/10.1159/000443167)

9 Stueven AK, Kayser A, Wetz C, Amthauer H, Wree A, Tacke F, Wiedenmann B, Roderburg C \& Jann H. Somatostatin analogues in the treatment of neuroendocrine tumors: past, present and future. International Journal of Molecular Sciences 201920 3049. (https://doi. org/10.3390/ijms20123049)

10 Khan S, Krenning EP, van Essen M, Kam BL, Teunissen JJ \& Kwekkeboom DJ. Quality of life in 265 patients with gastroenteropancreatic or bronchial neuroendocrine tumors treated with [177Lu-DOTA0,Tyr3] octreotate. Journal of Nuclear Medicine 2011 52 1361-1368. (https://doi.org/10.2967/jnumed.111.087932)

Received in final form 14 April 2021

Accepted 20 April 2021 